\title{
Analysis of Partial Discharge Patterns for Generator Stator Windings
}

\author{
Tae-Sik Kong, Hee-Dong Kim, Tae-Sung Park, Kyeong-Yeol Kim, Ho-Yol Kim \\ Korea Electric Power Corporation (KEPCO) Research Institute, Daejeon, South Korea
}

Email address:

kongts@kepco.co.kr (Tae-Sik Kong), hdkim@kepco.co.kr (Hee-Dong Kim), parkts@kepco.co.kr (Tae-Sung Park), k2yeol@kepco.co.kr (Kyeong-Yeol Kim), hoyolkim@kepco.co.kr (Ho-Yol Kim)

\section{To cite this article:}

Tae-Sik Kong, Hee-Dong Kim, Tae-Sung Park, Kyeong-Yeol Kim, Ho-Yol Kim. Analysis of Partial Discharge Patterns for Generator Stator Windings. American Journal of Electrical Power and Energy Systems. Vol. 4, No. 2, 2015, pp. 17-22. doi: 10.11648/j.epes.20150402.11

\begin{abstract}
Forced outage of generators due to stator winding insulation failure can result in significant financial loss because of the high cost repair and loss of production. In recent years, the demand for insulation diagnosis is increasing to prevent unexpected failures, as the capacity of generators has increased. Insulation diagnosis is composed of the insulation resistance measurement, polarization index measurement, dissipation factor (DF) tip-up test, AC current increasing ratio measurement, and the partial discharge (PD) measurement. In this paper, the results of the PD measurement and PD pulse pattern analysis performed on a healthy generator and two generators that experienced dielectric breakdown failure during operation is presented.
\end{abstract}

Keywords: Generator, Stator Winding, Insulation Failure, PD Pattern, AC Current, Dissipation Factor

\section{Introduction}

Defects in the insulation system of generator stator windings can be produced during manufacturing or due to thermal, mechanical, electrical, or chemical deterioration when the generator is operated for a long time. With insulation degradation due to a combination of such operating stresses, voids can be formed inside the insulation material, and dielectric breakdown may eventually result from partial discharge activity [1-3].

A forced outage of a generator during operation due to dielectric breakdown of the stator windings requires long repair time and hence results in enormous economic loss. Therefore, the importance of insulation diagnosis that evaluates the soundness of generator stator winding insulators is increasing. There exists a dielectric strength test method for verifying whether dielectric strength is sufficient by applying $2 \mathrm{E}+1 \mathrm{kV}$ ( $\mathrm{E}$ : rated voltage) for 1 minute and checking whether the insulation withstands the voltage without failing. However, such a test method is only used in special cases such as a shop test or an acceptance test for quality assurance of newly manufactured windings and not for maintenance testing of generators in the field. One of the methods for testing the dielectric strength for these generators is a high-potential test, which applies a voltage of
$1.25 \sim 1.5$ times the rated voltage for $1 \mathrm{~min}$, and in South Korea, this test method is only used in limited numbers of cases where the dielectric strength at failure or restoration is evaluated in the field [4-6]. Conventional dielectric tests for generator stator windings use a test voltage lower than the rated voltage and mainly use the insulation resistance measurement, polarization index (PI) measurement, $\mathrm{AC}$ current increasing ratio measurement, dissipation factor (DF) tip-up measurement, and partial discharge (PD) measurement [7-9].

Insulation diagnosis tests for generators stator winding with dielectric breakdown due to overheated copper conductors and abraded stator windings with induced external discharge noise of generators is carried out and presented in this paper. The PD patterns measured for normally deteriorated generators are analyzed, and the correlation between the causes of the defects and the PD patterns are shown.

\section{Experimental Procedure}

The polarization index (PI) was measured using a commercially available automatic insulation tester (Megger, $\mathrm{S} 1-5010$ ) at DC $5 \mathrm{kV}$ in individual phases before applying AC voltage to the stator windings. Commercially available equipment, namely, Schering bridge (Tettex Instruments), 
coupling capacitor, and PD detector (Tettex Instruments, TE 571), were used to measure $\mathrm{AC}$ current, dissipation factor, and PD magnitude, respectively. The Schering bridge consists of a high voltage (HV) supply (Type 5283), a bridge (Type 2818), and a resonating inductor (Type 5285). A HV supply and control system (Tettex Instruments, Type 5284), Schering bridge (Tettex Instruments, Type 2816), resonating inductor (Tettex Instruments, Type 5288), coupling capacitor, coupling unit, and PD detector were used to measure AC current, dissipation factor, and PD magnitude in $15 \mathrm{kV}$ generator stator windings. The HV supply and control system, Schering bridge, and resonating inductor were used to obtain the AC current and dissipation factor measurements. For PD measurements, AC voltage was applied to the generator stator winding through a connected HV supply and control system. The coupling capacitor (Tettex Instruments, 4,000 pF) amplified signals from the winding, which were sent to the coupling unit (Tettex Instruments, AKV 572) and then to the PD detector (Tettex Instruments, TE 571) that measures the magnitude and pattern of PD. The frequency band of the PD detector ranged from 40 to $400 \mathrm{kHz}$.

\section{Test results and Discussion}

\subsection{Failure Due to Copper Conductor Overheating}

This is a case where a large-scale water cooling steam turbine generator was tripped because of the destruction of the main insulation of the stator winding caused by cooling water supply discontinuance. The generator, which was manufactured with thermal class $\mathrm{B}$ insulation material with a maximum allowable temperature of $130{ }^{\circ} \mathrm{C}$, experienced a discontinuance of the cooling water supply. The discontinuance was caused by an error in the coolant supply system for approximately $10 \mathrm{~min}$ during normal operation, and the temperature of the stator windings increased rapidly to approximately $200{ }^{\circ} \mathrm{C}$. This caused the main insulation of the stator winding to fail, and a ground fault eventually tripped the generator. The melted compound of the connecting joint between the cooling water box and the stator winding caused by overheating is shown in Figure 1, and the failed insulation of the stator winding is shown in Figure 2.

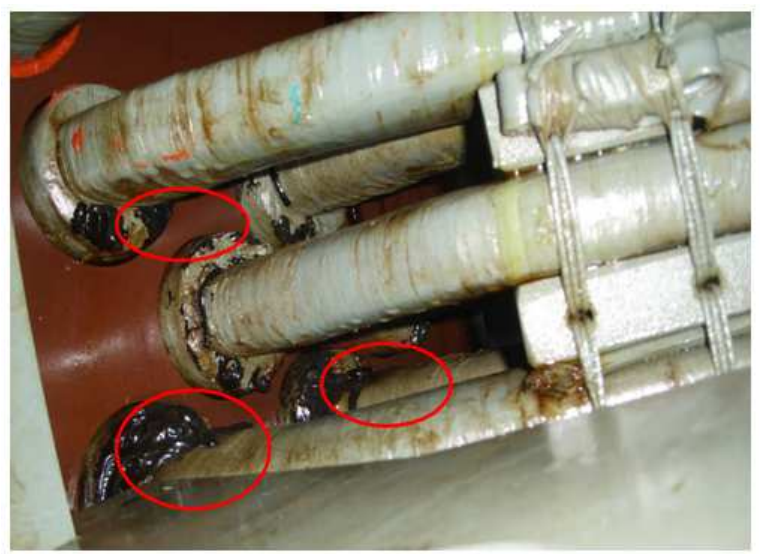

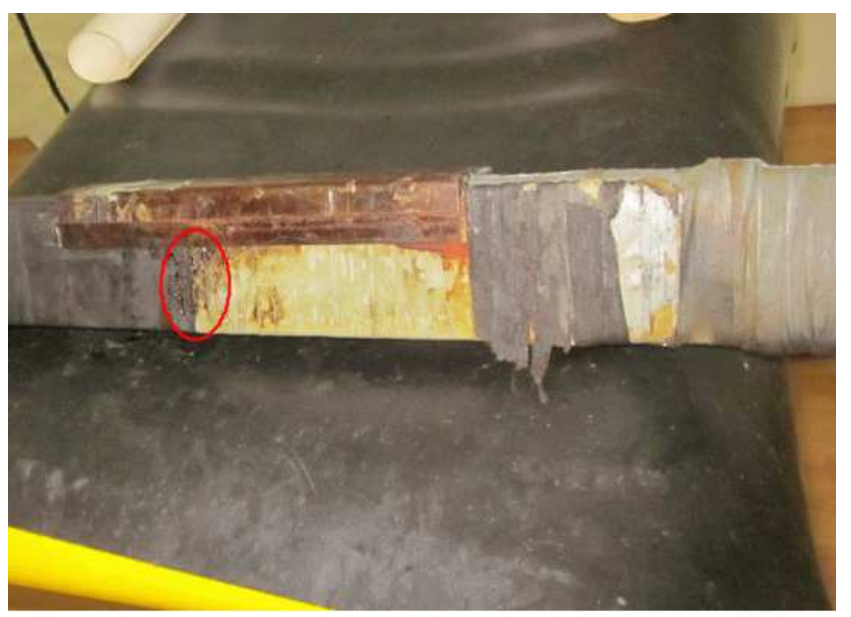

Figure 2. Stator winding insulation failure.

To check the insulation condition of the stator windings except for the bar with dielectric breakdown, the insulation resistance measurement, polarization index measurement, AC current increasing ratio test, dissipation factor tip-up measurement, and partial discharge measurement were performed. The AC current increasing ratio test consists of measurement of the increment in the current as the test voltage is increased, as shown in Figure 3. The dissipation factor tip-up test consists of measurement of the increment in the dissipation factor $(\tan \delta)$ with respect to its value at the initial test voltage, as shown in Figure 4. The AC current increasing ratio $(\Delta \mathrm{I})$ and dissipation increment factor $(\Delta \tan \delta)$ are closely related to the partial discharge. There is no partial discharge at low voltage, but partial discharge begins to occur at the void within the insulation system as the test voltage is increased, eventually increasing the values of the AC current and dissipation factor. Because these test measure the current and dissipation factor of the whole insulation system, it is used to find the average deterioration condition of the insulation material.

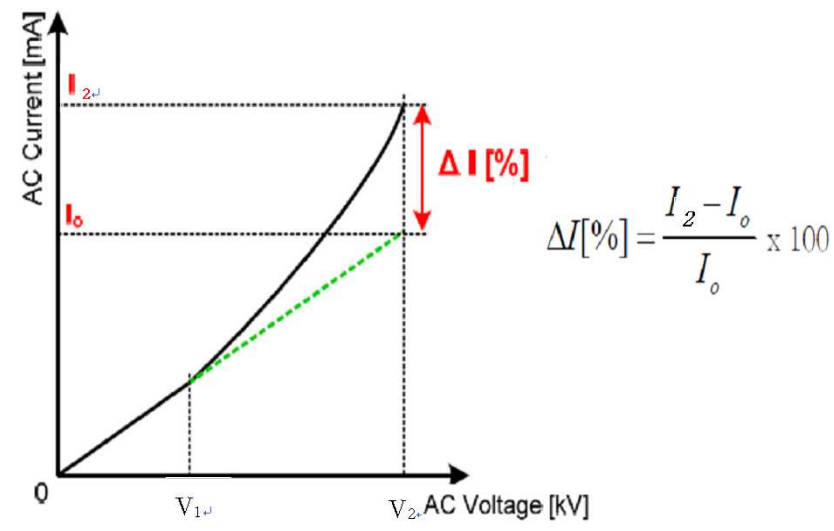

Figure 3. Voltage vs. AC current.

Figure 1. Compound melting due to overheating. 


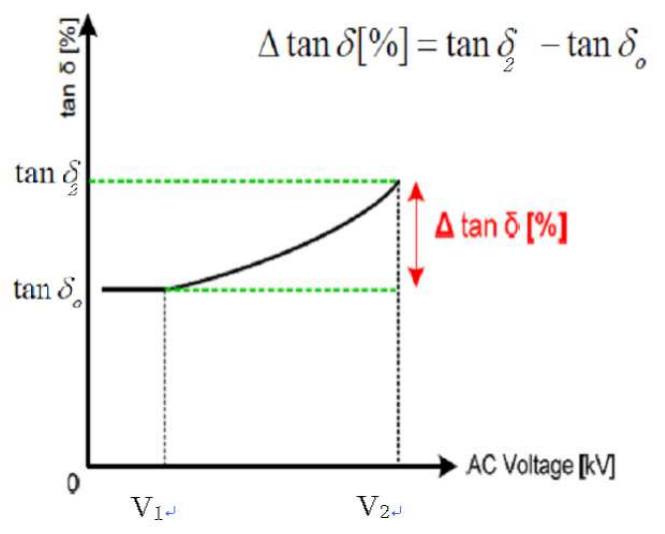

Figure 4. Voltage vs. Dissipation factor. voltage) for $\mathrm{AC}$ dielectric withstand voltage test, while holding this voltage for one minute. The $\mathrm{AC}$ current and dissipation factor were measured in $1 \mathrm{kV}$ increments as the test voltage was increased.

The AC current and DF measurement results at $15 \mathrm{kV}$ (phase voltage) were measured in two years prior to the failure. The same voltage of $15 \mathrm{kV}$ was applied to measure the values of $\Delta \mathrm{I}$ and $\Delta \tan \delta$ in order to compare the test results. The results show that both $\Delta \mathrm{I}$ and $\Delta \tan \delta$ increased after the failure, as shown in Table 1. This implies that PD started to occur more than two years prior to the failure, and also implies that the number of voids, which cause discharging within the insulation system, increased because of stator winding thermal overheating.

The line to line voltage of the generator was $26 \mathrm{kV}$, and test voltage was increased to $32.5 \mathrm{kV}$ (125\% of its line-line

Table 1. Results of AC current increasing ratio and dissipation factor Tip-Up measurements.

\begin{tabular}{lllll}
\hline \multirow{2}{*}{ Phases } & \multicolumn{2}{l}{ AC current $(\mathbf{\Delta I})[\boldsymbol{\%}]$} & \multicolumn{2}{l}{ Dissipation factor $(\boldsymbol{\Delta} \tan \delta)[\boldsymbol{\%}]$} \\
\cline { 2 - 5 } & Before failure & After failure & Before failure & After failure \\
\hline A & 1.79 & 3.08 & 1.05 & 1.27 \\
B & 2.14 & 2.08 & 0.97 & 1.09 \\
C & 2.06 & 2.08 & 1.08 & 1.26 \\
\hline
\end{tabular}

Table 2. PD measurement results.

\begin{tabular}{llll}
\hline \multirow{2}{*}{ Phases } & Before failure & After failure \\
\cline { 2 - 4 } & Discharge [pC] & Predominance & Discharge [pC] \\
\hline A & 7,100 & & 30,680 \\
B & 6,900 & $+P D \fallingdotseq-P D$ & 21,030 \\
C & 7,300 & & 29,770 \\
\hline
\end{tabular}

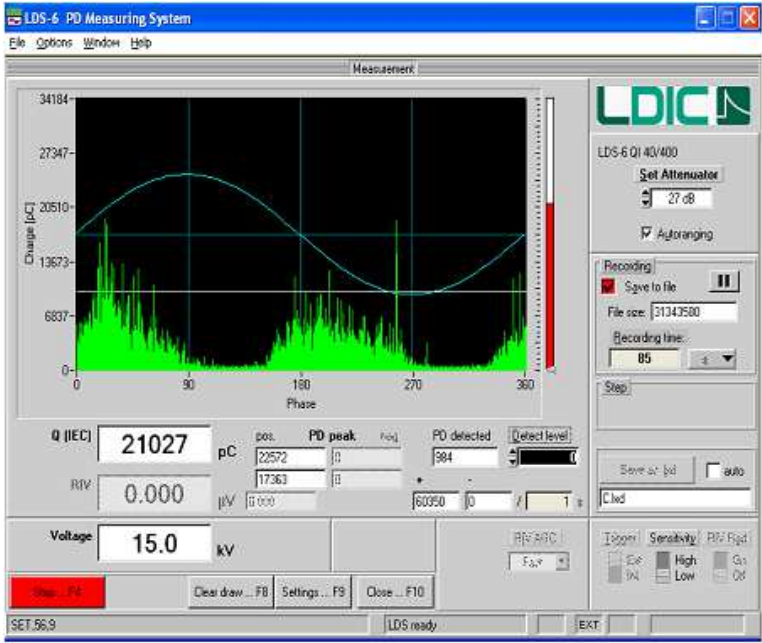

Figure 5. PD pattern for copper conductor overheating.

In the PD measurement, the maximum value of the multiple discharge pulses generated in the insulation system is measured. The largest discharge pulse is assumed to occur at the largest defect point, and the PD measurement is used to measure the level of PD activity in the largest defect in the insulation system. In the measurement, the voltage was 15 $\mathrm{kV}$ (phase voltage), and the results are shown in Table 2 . The

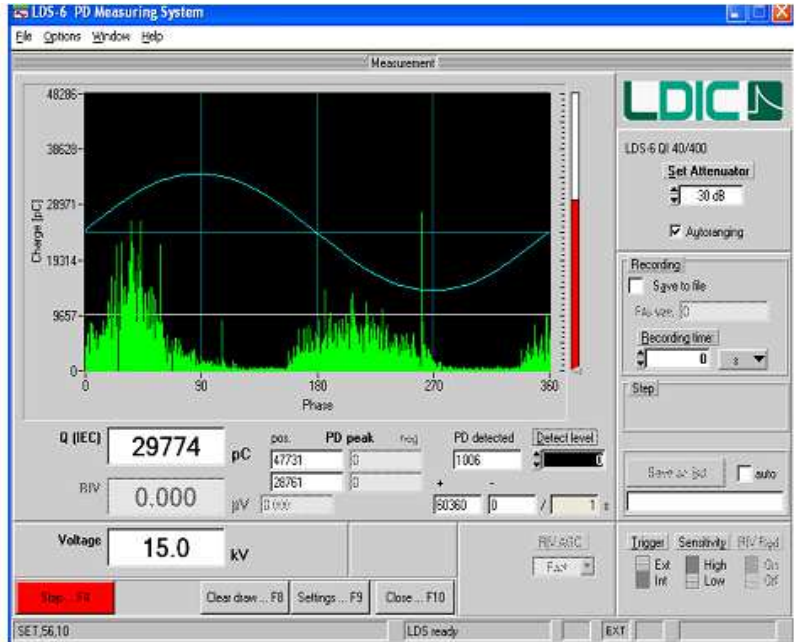

results show that the level of PD increased significantly after the failure and that there was also a change in the discharge pattern. Before the failure, the size and number of the negative discharge pulses, which appeared when the test voltage is positive, were similar to those of the positive discharge pulses, which appeared when the test voltage is negative. However, negative pulses were larger than the 
positive pulses after the failure due to thermal overheating, as shown in Figure 5. This pattern appears mainly in the gap between the copper conductor and strand insulation [10]. Furthermore, it was found that the insulation was not in good condition because the PD size was close to $30,000 \mathrm{pC}$ or greater [11].

This pattern is produced because of delamination between the copper conductor and the insulation because of the differential thermal expansion between the copper conductor and stator winding insulation as a result of rapid thermal overheating.

\subsection{Failure Due to Vibration on Stator Windings}

This is a case where a ground fault occurred on the stator winding of an air cooled gas turbine generator manufactured with the global vacuum pressure impregnation (VPI) method. The global VPI type generator was manufactured with the side ripple spring, which holds the stator winding in position inside the slot, removed. The stator winding was inserted into the slot and immersed in resin without the ripple spring to reduce the size of the core of the generator for saving manufacturing time and cost. This method is widely used in the generator industry, to keep the power density high in the globally competitive market.

However, when the generator is started and stopped, the winding insulation is subject to thermal expansion and contraction because of Joule heating, and the resin that holds the stator winding in its position within the slots is separated. This creates a gap between the winding and the slot, and the stator winding starts to vibrate at $120 \mathrm{~Hz}$ because of the electromagnetic force of the rotor, further reducing the thickness of the insulation. This increases the length subject to vibration, accelerating the wear process and eventually causing dielectric breakdown. In Figure 6, it can be observed how the semiconducting layer is almost eliminated because of the slot vibration-induced wear on the surface of the stator winding. The semi-conductive layer is used to reduce the partial discharge between the stator winding and the slot [12].

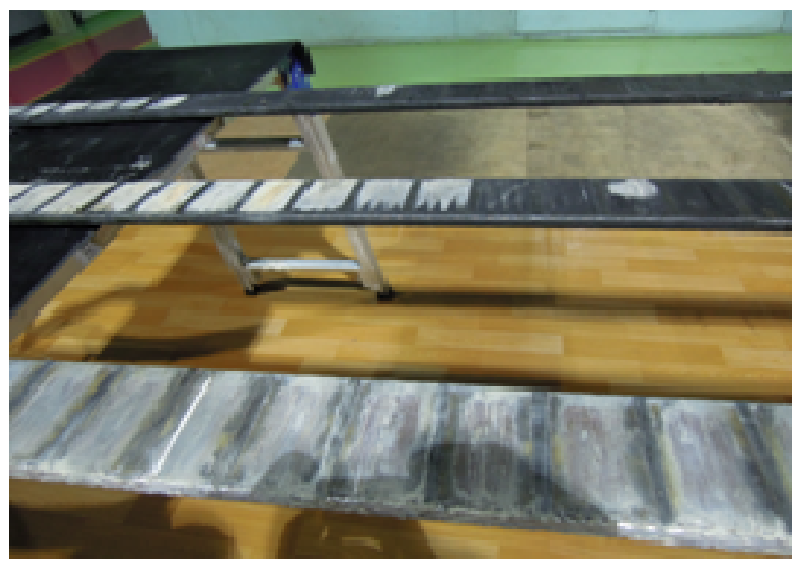

Figure 6. Wear in stator winding surface.

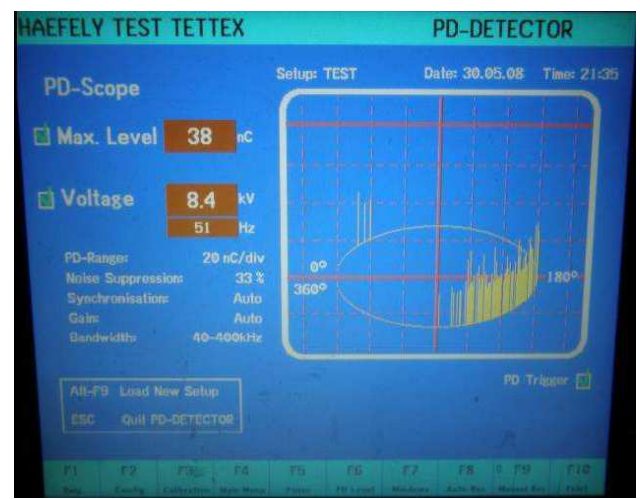

Figure 7. PD pattern for slot discharge.

Insulation diagnosis tests were performed on the remaining stator windings except for those that experienced dielectric breakdown. The PD measurement was carried out at rated phase voltage, and the measured PD magnitude and phase pattern is shown in Figure 7. The results show that the PD value was relatively high $(38,000 \mathrm{pC})$, and the PD pattern showed that the positive discharge pulses, which occurred at a test voltage phase angle of $225^{\circ}$, were produced more than negative discharge pulses, which occurred at $45^{\circ}$, forming a slot discharge pattern [8,9]. Most PDs are considered to occur at a gap between the stator winding and the slot, and it was also confirmed that the surface of the stator winding drawn from the slot was also significantly worn, as shown in Figure 6.

\subsection{Internal Discharge Pattern of Ground-Wall Insulation}

Figure 8 shows the PD measurements on a $22 \mathrm{kV}$ steam turbine generator, which has been operating for approximately 20 years. According to the test results, the location of the main discharge is considered to be in the void inside the ground-wall insulation because the positive and negative PDs occur almost similarly. This is because the voids within the ground-wall insulation were not completely removed during the vacuum pressure impregnation process of the winding manufacturing or because voids were created inside the ground-wall insulation as a result of thermal deterioration. This discharge pattern due to the internal voids of the insulation material occurs during normal deterioration and is a frequent form of partial discharge pattern.

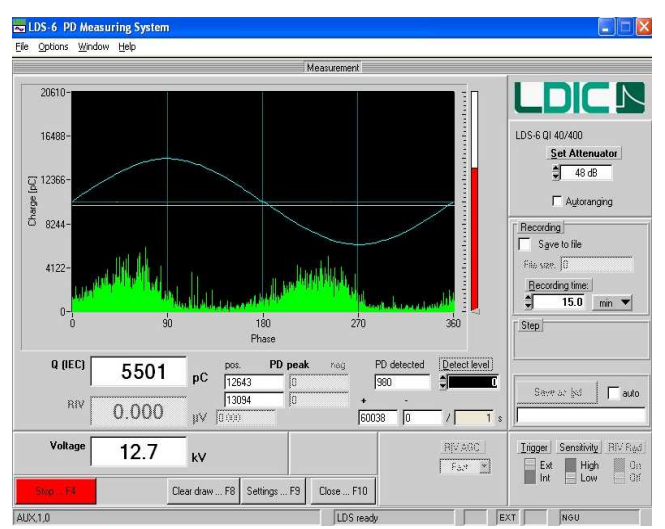

Figure 8. PD pattern for internal discharge. 


\subsection{Noise Occurrence Due to Gap Discharge}

The screen of the PD measurement during a PD test on a generator that failed because of overheating in the copper conductor is shown in Figure 9. The location of the preliminary pulses are near $0^{\circ}$ and $180^{\circ}$, and the size of the discharged pulses are almost identical. This pulse had a completely different shape with respect to the discharge pattern on insulation systems. This discharge pulse sizes being similar implies that the pulses occurred between particular electrodes. Because external noise pulses were detected in addition to the PD occurring at the electrical insulation, a visual inspection was performed to check the location that produced the noise. As a result, it was found that the contact between two isolated phase bus (IPB) bars, through which the generator output flowed for each phase, and the clip, which connected the two IPB bars to the common plate, was defective resulting in a discharge. This problem was rectified, and a test was conducted.

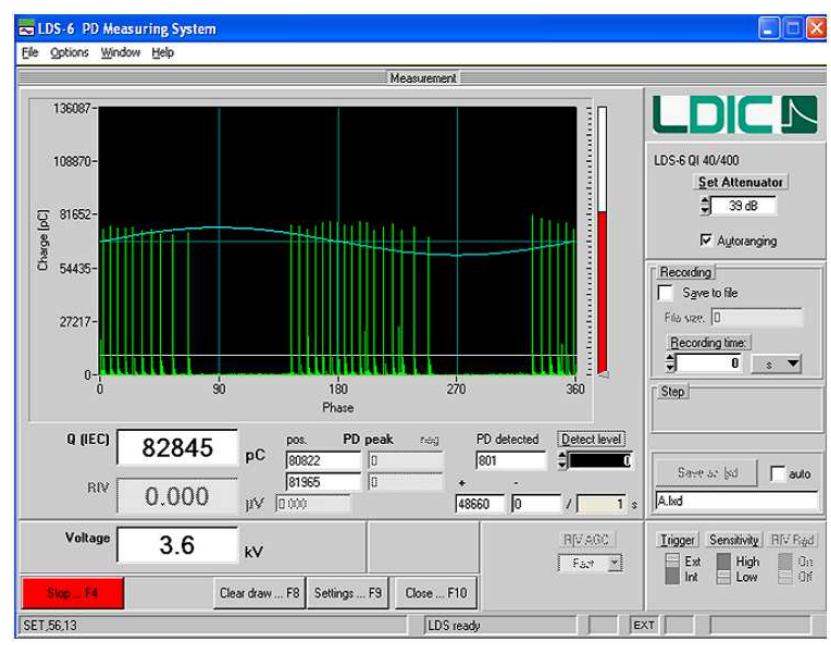

Figure 9. PD pattern for gap discharge noise.
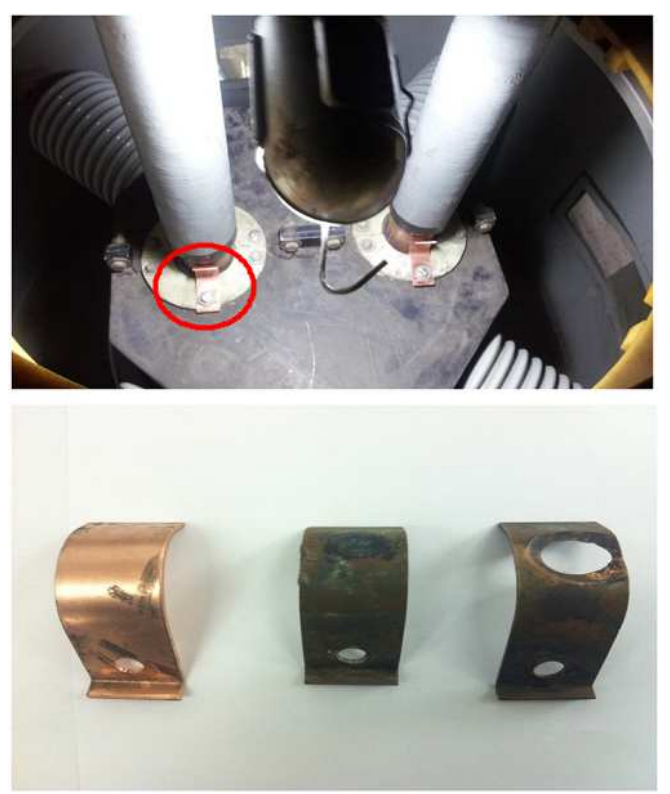

Figure 10. Gap discharge location.

\section{Conclusion}

This study conducted PD measurements on four cases, two generators that had dielectric breakdown, one generator undergoing normal deterioration process, and the other generator with external noise discharge, which have shown different discharge patterns. The PD patterns were analyzed and the correlation between the causes of the defects and the discharge patterns were presented.

First, the discharge pattern at the time of the dielectric breakdown due to overheating of the winding, which resulted from the loss of coolant for the stator winding, was examined. In this case, the negative pulses were larger and more frequent than the positive pulses. This was a result of a separation phenomenon occurring between the insulating material and copper conductor because of rapid differential thermal expansion between the copper conductor and insulation. Furthermore, when compared to the measurement performed prior to failure, the AC current increasing ratio, dissipation factor, and PD levels all increased, which subsequently led to a rapid deterioration.

Second, a generator manufactured according to the global VPI. method without a side ripple spring that immobilizes the stator winding in a slot was studied. Repeated expansion and contraction occurred when the generator stopped operating, creating a crack between the winding and slot. The generated vibration in the generator impaired the semiconducting layer, which primarily resulted in negative pulses.

Third, the PD pattern of the generator with normal temperature aging appeared to be very similar for positive and negative pulses.

Fourth, the level and status of the discharge pulses occurring in a particular external gap instead of insulation system of the generator appeared to be almost identical.

It was found that the discharge patterns differed depending on the location of the defects in the stator winding. Hence, an analysis of the PD patterns of a generator will be useful in maintenance control and operation according to type of defect.

\section{References}

[1] Hee-Dong Kim, "Analysis of Insulation Aging Mechanism in Generator Stator Windings", Journal of the KIEEME, Vol. 15, No2, pp. 119-126, 2002.

[2] R. Morin, R. Bartnikas and P. Menard, "A Three-Phase MultiStress Accelerated Electrical Aging Test Facility for Stator Bars", IEEE Trans. on Electrical Conversion, Vol. 15, No. 2, pp. $149 \sim 156,2000$.

[3] H. Zhu, C. Morton and S. Cherukupalli, "Quality Evaluation of Stator Coils and Bars under Thermal Cycling Stress", Conference Record of the 2006 IEEE International Symposium on Electrical Insulation, pp. $384 \sim$ 387, 2006.

[4] H. G. Sedding, R. Schwabe, D. Levin, J. Stein and B. K. Gupta, "The Role AC \& DC Hipot Testing in Stator Winding Ageing", IEEE Electrical Insulation and Electrical Manufacturing \& Coil Winding Conference, pp. 455 457, 2003. 
[5] "Recommended Practice for Insulation Testing of Large AC Rotating Machinery with High direct Voltage", New York : Institute of Electrical and Electronics Engineers, IEEE+ Std. 95-1977, pp. 13, 1977.

[6] IEEE Standard "IEEE Guide for Insulation Maintenance of Large Alternating-Current Rotating Machinery $(10,000 \mathrm{kVA}$ and Larger)" IEEE Std 56-1997, pp. 12, 1997.

[7] Hee-Dong Kim, Tae-Sik Kong, Young-Ho Ju, Byong-Han Kim "Analysis of Insulation Quality in Large Generator Stator Windings", Journal of Electrical Engineering \& Technology Vol. 6, No. 2, pp. 384-390, 2011.

[8] Claude Hudon and Mario Belec, "PD Signal Interpretation for Generator Diagnostics", IEEE Trans. on Dielectrics and Electrical Insulation, Vol. 12, No. 2, pp. 297 319, 2005.

\section{Author Profile}

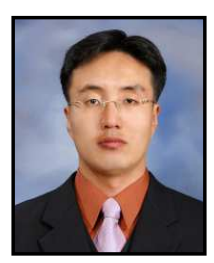

\section{Tae-Sik Kong}

He received his B.S. degree in Electrical Engineering from Chungbuk National University, Cheongju, Korea in 1997 and M.S. degree in Electrical Engineering from Chungnam National University, Daejeon, Korea in 2004. Since 1997, he has been working for the Korea Electric Power Corporation (KEPCO). He is currently a senior researcher with KEPCO Research Institute, Daejeon, Korea. His research interest is diagnostic test for rotating machine and transformer

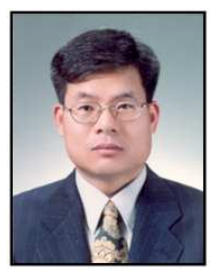

\section{Hee-Dong Kim}

He received the B.S., M.S., and Ph.D. degrees in Electrical Engineering from Hongik University, Seoul, Korea, in 1985, 1987, and 1998, respectively. Since 1990, he has been with Korea Electric Power Corporation (KEPCO) Research Institute, Daejeon, Korea, where he is currently a principal researcher. He was a visiting researcher with the Department of Electrical Engineering, Kyushu Institute of Technology, Kitakyushu, Japan. His research interests include aging mechanisms, diagnostic tests, partial discharge testing, and life assessment for rotating machines, and cable insulation systems.

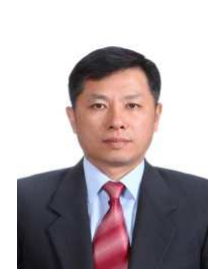

\section{Tae-Sung Park}

He received his B.S. degree in Chemistery from Gongju National University, Gongju , Korea in 2002. Since 1990, he has been working for the Korea Electric Power Corporation (KEPCO). He is currently a senior researcher with KEPCO Research Institute, Daejeon, Korea. His research interest is diagnostic test for rotating machines.
[9] Y. Ikeda and H. Fukagawa, ""A Method for Diagnosing the Insulation Deterioration in Mica-Resin Insulated Stator Windings of Generator"', Yokosuka Research Laboratory Rep. No. W88046, 1988

[10] IEEE Standard "Trial-Use Guide to the Measurement of Partial Discharge in Rotaing Machinery", IEEE Std 14342000, pp. 40, 2000

[11] H. Yoshida and U. Umemoto, "Insulation Diagnosis for Rotating Machine Insulation", IEEE Trans. on Electric Insulation, Vol. EI-21, No. 6, pp. 1021-1025, 1986

[12] J.H. Dymond, N. Stranges, K. Younsi and J. E. Hayward, "Stator Winding Failures : Contamination, Surface Discharge, Tracking", IEEE Trans. on Industry Applications, Vol. 38, No. 2, pp. 577-583, 2002.

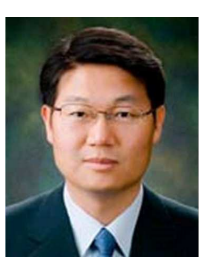

\section{Kyeong-Yeol Kim}

$\mathrm{He}$ received his B.S. degree in Electrical Engineering from Chonbuk National University, Jeonju, Korea in 1994 and M.S. degree in Electrical Engineering from Chonbuk National University, Jeonju, Korea in 1996. Since 1997, he has been working for the Korea Electric Power Corporation (KEPCO). He is currently a senior researcher with KEPCO Research Institute, Daejeon, Korea. His research interest is failure analysis of electrical machines in power plant.

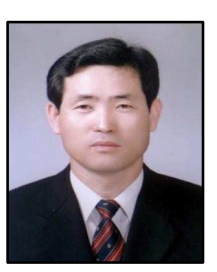

\section{Ho-Yol Kim}

$\mathrm{He}$ received M.S degrees in Information Engineering from Inha University, Incheon, Korea, in 1990. Since 1995, after starting his work in Korea Electric Power Corporation (KEPCO) in 1977, he has been working primarily on power plant control as a specialist and researcher in KEPCO head quarter and research institute respectively. His major interests are research on control system tuning and partial discharge in electrical devices as well. Currently, he is the Director of Technology Support Centre in KEPCO Research Institute where the researchers work in 4 teams. 